\title{
Inpatient Dermatology Best Practice Strategies for Educating and Relaying Findings to Colleagues
}

\author{
Adam Faletsky ${ }^{1,2}$ (D) - Jane J. Han ${ }^{1,3} \cdot$ Arash Mostaghimi $^{1}$ \\ Accepted: 16 October 2020 / Published online: 27 October 2020 \\ (C) Springer Science+Business Media, LLC, part of Springer Nature 2020
}

\begin{abstract}
Purpose of Review This review examines the role of education and relaying findings to non-dermatologist colleagues when performing inpatient dermatology consults. We highlight best practices for communication and education.

Recent Findings Non-dermatologists receive minimal training on the diagnosis and the management of skin conditions. Efforts to teach dermatology in the inpatient setting via traditional didactics have been met with limited success, and hospitalists have indicated a desire to learn from specialists. Incorporating education into standard consultation practices including the note, oneon-one communication, and bedside rounds can efficiently improve teaching and patient care.

Summary Our key principles of consultation emphasize communication, use of pre-existing components of a consultation to teach, and close follow-up. Inpatient dermatologists can implement these simple but effective measures to encourage education and communication with primary teams for both in-person and telehealth consults.
\end{abstract}

Keywords Inpatient dermatology · Dermatology consults · Dermatology education · Dermatology communication $\cdot$ Relaying findings $\cdot$ Teledermatology

\section{Introduction}

Inpatient consultation by board-certified dermatologists has been shown to improve outcomes in patients with a range of dermatologic illness, with $58-96 \%$ of consults leading to changes in patient treatment plans [1]. In addition to supporting patient care, inpatient dermatology consultants play an essential role in educating the primary team. Although not explicitly a part of the dermatology curricula, the ability to teach while relaying information to our colleagues is a core competency of any inpatient consultative

Adam Faletsky and Jane J. Han contributed equally to this work.

This article is part of the Topical Collection on Hospital-Based Dermatology

Arash Mostaghimi

amostaghimi@bwh.harvard.edu

1 Department of Dermatology, Brigham and Women's Hospital, 221 Longwood Avenue, Boston, MA 02115, USA

2 School of Medicine, Tufts University, Boston, MA, USA

3 Stritch School of Medicine, Loyola University Chicago, Maywood, IL, USA service. This education is often informal and is commonly integrated into the consultant's daily responsibilities.

Non-dermatologists' training in skin pathology is limited, and the measures to teach common dermatologic conditions through traditional didactics have been limited in efficacy [2, 3]. In contrast, point-of-care teaching at the bedside has been demonstrated to improve educational outcomes and patient satisfaction $[4,5]$. In this review, we examine the best practices in inpatient dermatology for educating and relaying findings to colleagues.

\section{Communication}

The initial interactions with the primary team determine the tone of the entire consultation. Although the consult question may not be fascinating and the timing may not be ideal, remember that you are being consulted for one of three reasons: (1) the person who is calling does not understand what is happening to the patient, (2) the person who is calling is scared, or (3) somebody is making the person who is calling (i.e. a trainee) ask for a consult. As clinicians, we have been on both sides of all three scenarios, and approaching this first interaction with a spirit of understanding can help develop trust. 
After receiving the initial consult page and doing a brief chart review, you should promptly communicate directly with the primary team-introduce yourself and allow your colleague to tell you about the patient. While answering services or electronic messaging can be used for demographic intake, there is no substitution for direct communication. In this conversation, remember the three guidelines for medical educators proposed by Dr. Daniel Federman: think out loud, stick to the basics, and be kind [6].

Think out Loud On your call, work with the team to clearly define the consult question. Be patient-it can be difficult for requesting teams to articulate a clear clinical question for a consult, especially if they are not familiar with the condition they are calling about [7]. When you ask clarifying questions, spend an extra moment to explain why you are asking those specific questions by providing a background on your thought process [8]. Apply a similar approach to requesting additional laboratory tests or imaging studies. This simple practice will help educate the team and lay a foundation for understanding upcoming recommendations [9].

Stick to the Basics The diagnostic approach in dermatology is fundamentally different from other fields of medicine: while most other fields of medicine deduce differentials from histories, dermatologists gain most of their information from the skin exam. Therefore, the education of our non-dermatology colleagues requires a systematic approach. Instead of focusing on rare conditions with long, multisyllabic, Latin names, or worse, unexplained acronyms, starting with common dermatologic conditions that may be familiar to the primary team can help build on other's experience and perspective [10•].

A simplified breakdown of lesion morphology supplements and supports your differential and serves as a scaffold for the primary team's future care of dermatologic patients. Other pragmatic teaching points include fundamental approaches to pharmacotherapy such as steroid class and vehicle. As specialists, knowledge and concepts that we find to be routine are often novel and educational for primary teams. Reiterating teaching points in a progress note reinforces key findings and recommendations.

Be Kind As consultants, we must assume the best intent when talking to the primary team. We are colleagues, who should be patient in our interactions, reminding ourselves that consulting teams are asking for help. Curt exchanges with the primary team may gain you short-term efficiency but could be interpreted as indifference or pushback (refusing the consult), a common complaint regarding consultants [11•]. A potential reason for perceived pushback may stem from suboptimal communication skills, which impact our ability to clearly relay findings and recommendations to the primary team [12]. A consultant can prevent miscommunication by explicitly expressing willingness to help and immediately agreeing to the consult request $[7,13]$. The relationships we build during consults are critical for future collaborations between dermatologists and other fields and help ensure that the patients receive appropriate care.

\section{Best Practices for Teaching}

Although it may feel out of place to teach during a busy period of patient care, most hospitalists indicate a desire to learn from consultants and believe that the responsibility of initiating the educational encounter falls on the consultant [11•].

Teaching Starts with the Note Notes are a convenient way to educate primary teams. Good notes are succinct yet thorough, beginning with the consult question, followed by the pertinent history, physical, differential, and recommendations. A thorough differential is warranted along with an explanation of why certain diagnoses are less likely to help walk the primary team through your decision-making.

A note can be educational without being excessively long. You should clearly specify your recommendations, ideally in bullets or numbers, at the bottom of the note (Fig. 1). Although there is tremendous educational value in the note, allowing busy teams to rapidly extract precise conclusions and tasks from your notes is critical to the workflow. Nobody wants to be greeted by a wall of text when trying to wrap up patient care on a busy day.

Direct Teaching Is the Best Way Direct communication is the most effective method of educating colleagues about patient management. Consultants should search for teaching opportunities during the consult follow-up, where most education of the primary team occurs [7]. Primary teams who feel comfortable with their consultants are more likely to engage in a conversation that facilitates a deeper understanding of the consult team's reasoning [8]. This does not need to be a lengthy process - a two to three minute conversation in the workroom on your way off the floor can do wonders for collegiality, coordination of care, and education. Dermatologists should make every effort to attend teaching rounds, morbidity and mortality conferences, and any other teaching conferences where cases they have consulted on the inpatient service are being discussed.

Bedside Teaching Bedside rounds are the gold standard for learning in the field of dermatology, which emphasize honing observational skills by describing lesion morphologies $[4$, $10 \bullet$. With experiential learning, our colleagues can learn how to diagnose dermatologic conditions while receiving real-time feedback at the bedside. This method of teaching allows non-dermatologists to develop their clinical dermatology skills, recognize details in skin lesions, and improve their 
Dermatology consult note $6 / 13 / 2019$

Consulting team: Internal Medicine

Requesting provider: Dr. G

Reason for consult: Concern for SJS and wound care recommendations

1. Clear consult request.

Hospital course:

AG is a $34 \mathrm{y} / 0$ male with history of new onset epilepsy, TIIDM, and obesity transferred from an outside hospital on $6 / 13$. Patient presented to an outside hospital on $6 / 13$ for progressively worsening rash that began on the face on $6 / 9$. The rash subsequently spread to trunk and extremities over the course of 2 days. Associated symptoms include headache, subjective fever, dyspnea that began around the same time as the rash. Of note, patient was started on phenytoin by her neurologist 5 weeks prior. Patient was admitted to floor and dermatology consulted for further management.

Physical exam findings:

Images in Media tab were reviewed and patient was also examined in person.

Vitals: 38.6 C/ BP:97/65/ P: 88/ RR: 18

Well appearing female in no acute distress.

Central facial erythema and edema, diffuse morbilliform light pink rash on chest, bilateral upper and lower extremities with sparing of the palms and soles. No evidence of mucosal or genital involvement, or desquamation.

Mild head and neck adenopathy.

No extremity edema appreciated.

Additional photos in Media tab.

Key laboratory findings:

CBC with dif: WBC 12.2 / Eosinophils $20 \%$ / Atypical lymphocytes $1 \%$

BMP within normal limits: BUN $8 \mathrm{mg} / \mathrm{dL} / \mathrm{Cr} 0.98 \mathrm{mg} / \mathrm{dL}$

LFTs: ALT $151 \mathrm{U} / \mathrm{L} /$ AST $171 \mathrm{U} / \mathrm{L}$

\section{Assessment: $34 \mathrm{y} / 0$ male with likely DRESS syndrome}

Given the patient's recent initiation of phenytoin and onset of an acute morbilliform rash with facial swelling in the context of 1) facial swelling, 2) eosinophilia, 3) atypical lymphocytosis, and 4) inflammation of the liver, drug reaction with eosinophilia and systemic symptoms (DRESS) is currently highest on our differential. Additionally, systemic finding such as patient's fever, headache, and dyspnea also support a diagnosis of DRESS. DRESS has variable presentations which may affect one or more organ systems such as cardiopulmonary, renal, and hepatic and lab monitoring to assess for their involvement is important. The timing of the rash, associated systemic symptoms, and lack of mucosal membrane involvement argues against other severe drug reactions (e.g. SJS/TEN).

Recommendations:

- Please stop phenytoin and work with neurology to transition to another epilepsy treatment.

- Biopsy is not indicated at this time-DRESS is a clinical diagnosis.

- Please obtain daily laboratory testing of LFTs, $\mathrm{Cr} / \mathrm{BUN}$, and CBC with differential to monitor progression.

- Begin oral prednisone $50 \mathrm{mg}$. We will initiate taper based on clinical response.

- Once the rash starts to show signs of improvement, and normalization of LFTs, eosinophilia, and atypical lymphocytes, decrease by $10 \mathrm{mg}$ every 2 weeks

- Please contact the inpatient dermatology team before discharge so we may set up outpatient appointment. Follow up will be important as DRESS syndrome may have autoimmune sequelae that requires close monitoring.

- Clinic number: $1(800)$-GOT-RASH

Please page me with any additional questions $\# 0000$.

Resident J

Dermatology resident, PGY-3

Fig. 1 A demonstration of the key components that make a consultation note clear and helpful, while incorporating teaching points

ability to make accurate diagnoses [14]. In addition to teaching the primary team, this approach simultaneously educates the patient and ensures a collective understanding of the plan.

Despite these benefits, bedside rounds have become less common over time, largely due to time constraints and increased reliance on electronic medical records for patient care and communication [15]. For dermatology consultants who may be seeing ambulatory patients off site, bedside rounds pose additional logistic challenges when trying to coordinate with primary teams. In the absence of formal bedside rounds, encouraging any available members of the primary team (from medical student to attending) to join you when you are doing your evaluation is beneficial. The limited exposure of most physicians to dermatology has been well documented, and 
efforts to improve dermatology education are underway, including dedicated electives, specialized didactics, and computer modules [16]. Despite this progress, the most effective teaching remains to be the direct, point-of-care discussions of patient care.

\section{Consistent Follow-Up and Sign-Off}

After the consult, routine follow-up with the patient and the team will help maintain insight into the patient, the ongoing communication with the team, and the potential for further educational opportunities. On some occasions, you may find that your recommendations may not be followed-discuss with the primary team to determine if this is a result of miscommunication or an active decision of the primary team after input from the patient and other consultants. Once the patient is stable, communicate with the primary team before signing off to close the loop of communication and to make sure all outstanding tests and follow-ups are accounted for.

\section{Teledermatology Consults}

The steady growth of teledermatology has turned into a meteoric boom in the context of the COVID pandemic [17•, 18]. Although practice habits have begun to normalize, the increased acceptance and the use of teledermatology, whether formal or informal, is likely to become a mainstay of inpatient dermatology consults moving forward. Most teledermatology consults utilize store-and-forward technology, in which images, videos, and pertinent medical information are collected from the patient by a trained practitioner and sent to a dermatologist at an off-site location. In these cases, the primary team is responsible for the initial data gathering on behalf of the dermatology team.

Similar to a face-to-face consult, the key to a successful consultation via telemedicine is contingent on the strong communication with the primary team. Given that the primary team is now your eyes and ears, teledermatology lends itself naturally to education - when you ask for additional photos or data points, remember the key teaching principles illustrated above (be kind, think out loud, and stick to the basics) to maximize educational value (Table 1). As teledermatology becomes more commonplace in the inpatient setting, we must continue to uphold the importance of educating our peers on the dermatological principles that can be learned from each new patient case.

\section{Conclusion}

Although dermatology is perceived to be a predominantly outpatient specialty, dermatologists serve an important role in the inpatient setting. In addition to benefiting patient care, dermatology consultants are essential contributors to education for non-dermatology trainees and attending physicians. Clear communication and consistent education will simultaneously improve both the care of the patient of record and the care of future patients. While direct teaching is ideal, education most often begins with the note, in which dermatologists can systematically outline their diagnostic thought process for

Table 1 Key concepts in communication and education

\section{Communication}

Think out loud

Stick to the basics

Be kind

Teaching

Teaching starts with the note

Direct teaching is the best way

Bedside teaching

Follow-up

Teledermatology
Help the primary team develop a consult question while providing context and educational cues.

"It sounds like you need assistance determining what the cause of the patient's lesion is. Has she recently started a new medication? The reason I ask is..."

Teach high-yield information in line with the consulting team's respective specialty.

"The most likely differential for this pediatric patient's diffuse erythroderma and desquamative rash with bullae is likely staph scalded skin syndrome, but we should also consider other bullous diseases and drug reactions. With rashes like this, it's helpful to first determine if the bullae is tense or flaccid because it can help distinguish between..."

Take the time to develop a collegial relationship with each team you work with.

"We would be more than happy to go see this patient. Feel free to page me if there are any questions or concerns."

Strike a balance between being thorough and efficient.

See Fig. 1.

Try to communicate with the primary team in person, but if that is not possible, telephone communication is superior to a page or the EMR.

The best way to teach is to look at the skin and discuss the plan with the primary team and the patient. 
the primary team. If time permits, bedside rounds take advantage of experiential learning and allow our non-dermatologist colleagues to gain fundamental dermatological skills in real time. As inpatient consults evolve to incorporate telemedicine, dermatologists should continue to focus on appropriate communication and education in all settings.

Author Contributions All authors made substantial contributions to the conception or design of the work. AF and JH performed the literature search. All authors drafted the work, revised it critically for important intellectual content, approved the version to be published, and agree to be accountable for all aspects of the work.

\section{Compliance with Ethical Standards}

Conflict of Interest Financial interests: Dr. Mostaghimi reports personal fees from Pfizer, Hims, and 3Derm and holds equity in Hims and Lucid.

Human and Animal Rights and Informed Consent This article does not contain any studies with human or animal subjects performed by any of the authors.

\section{References}

Papers of particular interest, published recently, have been highlighted as:

\section{- Of importance}

1. Madigan LM, Fox LP. Where are we now with inpatient consultative dermatology?: assessing the value and evolution of this subspecialty over the past decade. J Am Acad Dermatol. 2019;80(6): 1804-8. https://doi.org/10.1016/j.jaad.2019.01.031.

2. McCleskey PE, Gilson RT, DeVillez RL. Medical student core curriculum in dermatology survey. J Am Acad Dermatol. 2009;61(1):30-35.e4. https://doi.org/10.1016/j.jaad.2008.10.066.

3. Beshay A, Liu M, Fox L, Shinkai K. Inpatient dermatology consultative programs: a continued need, tools for needs assessment for curriculum development, and a call for new methods of teaching. $\mathrm{J}$ Am Acad Dermatol. 2016;74(4):769-71. https://doi.org/10.1016/j. jaad.2015.11.017.

4. Gonzalo JD, Heist BS, Duffy BL, Dyrbye L, Fagan MJ, Ferenchick $\mathrm{G}$, et al. The art of bedside rounds: a multi-center qualitative study of strategies used by experienced bedside teachers. J Gen Intern Med. 2013;28(3):412-20. https://doi.org/10.1007/s11606-0122259-2.

5. Monash B, Najafi N, Mourad M, et al. Standardized attending rounds to improve the patient experience: a pragmatic cluster randomized controlled trial. J Hosp Med. 2017;12(3):143-9. https:// doi.org/10.12788/jhm.2694.

6. Thibault G, Katz JT, Arky R. DANIEL D. FEDERMAN: 19282017. Trans Am Clin Climatol Assoc 2018;129:xcviii-c.

7. Miloslavsky EM, McSparron JI, Richards JB, Puig A, Sullivan AM. Teaching during consultation: factors affecting the resident- fellow teaching interaction. Med Educ. 2015;49(7):717-30. https:// doi.org/10.1111/medu.12760.

8. Pavitt S, Bogetz A, Blankenburg R. What makes the "perfect" inpatient consultation? A qualitative analysis of resident and fellow perspectives. Acad Med. 2020;95(1):104-10. https://doi.org/10. 1097/ACM.0000000000002867.

9. Lee T, Pappius EM, Goldman L. Impact of inter-physician communication on the effectiveness of medical consultations. Am J Med. 1983;74(1):106-12. https://doi.org/10.1016/0002-9343(83)911269.

10. Afifi L, Shinkai K. Optimizing education on the inpatient dermatology consultative service. Semin Cutan Med Surg. 2017;36(1): 28-34. https://doi.org/10.12788/j.sder.2017.003 Importance: This article highlights how dermatology can be taught in the hospital setting and elaborates on both direct learning through patient care and indirect learning to enhance a trainee's dermatology skills.

11. Adams TN, Bonsall J, Hunt D, et al. Hospitalist perspective of interactions with medicine subspecialty consult services. J Hosp Med. 2018;13(5):318-23. https://doi.org/10.12788/jhm.2882 Importance: This study demonstrated that hospitalists at the receiving end of the teaching interaction are willing to be taught.

12. Gupta S, Alladina J, Heaton K, Miloslavsky E. A randomized trial of an intervention to improve resident-fellow teaching interactions on the wards. BMC Med Educ. 2016;16(1):276. Published 2016 Oct 20. https://doi.org/10.1186/s12909-016-0796-9.

13. Winn AS, Stafford DEJ, Miloslavsky EM, McSparron JI, Grover AS, Boyer D. Making the consult interaction more than a transaction: helping fellows be better teachers and residents be better learners. J Pediatr. 2019;209:3-4.e2. https://doi.org/10.1016/j. jpeds.2019.04.003.

14. Braverman IM. To see or not to see: how visual training can improve observational skills. Clin Dermatol. 2011;29(3):343-6. https://doi.org/10.1016/j.clindermatol.2010.08.001.

15. Gonzalo JD, Chuang CH, Huang G, Smith C. The return of bedside rounds: an educational intervention. J Gen Intern Med. 2010;25(8): 792-8. https://doi.org/10.1007/s11606-010-1344-7.

16. Rourke L, Oberholtzer S, Chatterley T, Brassard A. Learning to detect, categorize, and identify skin lesions: a meta-analysis. JAMA Dermatol. 2015;151(3):293-301. https://doi.org/10.1001/ jamadermatol.2014.3300.

17. Rismiller K, Cartron AM, Trinidad JCL. Inpatient teledermatology during the COVID-19 pandemic. J Dermatolog Treat. 2020;31(5): 441-3. https://doi.org/10.1080/09546634.2020.1762843 Importance: The COVID-19 pandemic is a shifting point for dermatologists as teledermatology becomes mainstream. This study demonstrates teledermatology's rapid implementation into our medical system.

18. Perkins S, Cohen JM, Nelson CA, Bunick CG. Teledermatology in the era of COVID-19: experience of an academic department of dermatology. J Am Acad Dermatol. 2020;83(1):e43-4. https://doi. org/10.1016/j.jaad.2020.04.048.

Publisher's Note Springer Nature remains neutral with regard to jurisdictional claims in published maps and institutional affiliations. 